\title{
Axionic band topology in inversion-symmetric Weyl-charge-density waves
}

\author{
Benjamin J. Wieder $\odot,{ }^{1,2,3,{ }^{*}}$ Kuan-Sen Lin ${ }^{4}$ and Barry Bradlyn $\odot^{4, \dagger}$ \\ ${ }^{1}$ Department of Physics, Massachusetts Institute of Technology, Cambridge, Massachusetts 02139, USA \\ ${ }^{2}$ Department of Physics, Northeastern University, Boston, Massachusetts 02115, USA \\ ${ }^{3}$ Department of Physics, Princeton University, Princeton, New Jersey 08544, USA \\ ${ }^{4}$ Department of Physics and Institute for Condensed Matter Theory, University of Illinois at Urbana-Champaign, \\ Urbana, Illinois 61801-3080, USA
}

(Received 29 April 2020; accepted 14 September 2020; published 14 October 2020)

\begin{abstract}
In recent theoretical and experimental investigations, researchers have linked the low-energy field theory of a Weyl semimetal gapped with a charge-density wave (CDW) to high-energy theories with axion electrodynamics. However, it remains an open question whether a lattice regularization of the dynamical Weyl-CDW is in fact a single-particle axion insulator (AXI). In this Rapid Communication, we use analytic and numerical methods to study both lattice-commensurate and incommensurate minimal (magnetic) Weyl-CDW phases in the mean-field state. We observe that, as previously predicted from field theory, the two inversion $(\mathcal{I})$-symmetric Weyl-CDWs with $\phi=0, \pi$ differ by a topological axion angle $\delta \theta_{\phi}=\pi$. However, we crucially discover that neither of the minimal Weyl-CDW phases at $\phi=0, \pi$ is individually an AXI; they are instead quantum anomalous Hall (QAH) and "obstructed" QAH insulators that differ by a fractional translation in the modulated cell, analogous to the two phases of the Su-Schrieffer-Heeger model of polyacetylene. Using symmetry indicators of band topology and non-Abelian Berry phase, we demonstrate that our results generalize to multiband systems with only two Weyl fermions, establishing that minimal Weyl-CDWs unavoidably carry nontrivial Chern numbers that prevent the observation of a static magnetoelectric response. We discuss the experimental implications of our findings and provide models and analysis generalizing our results to nonmagnetic Weyl- and Dirac-CDWs.
\end{abstract}

DOI: 10.1103/PhysRevResearch.2.042010

In condensed matter physics, one of the most important tools is low-energy field theory. From the $\mathbf{k} \cdot \mathbf{p}$ Hamiltonian of a solid-state material, one can develop an effective action to characterize robust, and frequently topological, longwavelength response effects [1-9]. However, to extrapolate from a low-energy field theory to an experimentally observable response, one must carefully complete the theory to short (UV) wavelengths - specifically, two field theories that are identical at the $\mathbf{k} \cdot \mathbf{p}$ level may differ at large momenta, leading to distinct physical interpretations. For example, $\mathcal{H}(\mathbf{q})=$ $\sigma^{x} q_{x}+\sigma^{y} q_{y}$ can characterize one of the twofold Dirac points in a graphenelike two-dimensional (2D) semimetal [10-12] or the isolated Dirac point on the surface of a time-reversal$(\mathcal{T}$-) symmetric 3D topological insulator (TI) [13-16]. While $\mathcal{H}(\mathbf{q})$ always carries the (Hall) response of a half-level $(2+$ 1)D Chern-Simons theory $[4,5,13,14,17]$, the total response depends on the UV completion, which includes either compensating Dirac points in 2D [4,17-26] or the nontrivial bulk of a $3 \mathrm{D}$ TI $[4,5,13,14,17,25-31]$.

\footnotetext{
*bwieder@mit.edu

†bbradlyn@illinois.edu
}

Published by the American Physical Society under the terms of the Creative Commons Attribution 4.0 International license. Further distribution of this work must maintain attribution to the author $(s)$ and the published article's title, journal citation, and DOI.
Some of the most intriguing low-energy field theories involve condensed-matter realizations of high-energy electrodynamics [6]. In 3D insulators, the long-wavelength response is governed by the action

$$
S\left[A_{\mu}\right]=\frac{1}{4 \pi^{2}} \int d^{4} x \epsilon^{\mu \nu \lambda \rho}\left(\theta \partial_{\mu}+v_{\mu}\right) A_{\nu} \partial_{\lambda} A_{\rho},
$$

in which $A_{\mu}$ is the electromagnetic gauge potential [2,4,5] [32] and $v_{\mu}$ is a rotational-symmetry-breaking vector that determines the quantum anomalous Hall (QAH) response. When $v_{\mu}=(0, \mathbf{v})$ is constant,

$$
\sigma_{i j}^{H}=\frac{e^{2} v_{k}}{h} \epsilon_{k i j},
$$

where $\sigma_{i j}^{H}$ is the Hall conductivity tensor. For gapped periodic systems,

$$
\mathbf{v}=C_{i}\left(\mathbf{k} \cdot \mathbf{R}_{i}\right) \mathbf{G}_{i},
$$

where $\mathbf{R}_{i}$ is a primitive lattice vector, $\mathbf{G}_{i}$ is a primitive reciprical lattice vector, and $C_{i}\left(\mathbf{k} \cdot \mathbf{R}_{i}\right) \equiv v_{i} /\left|\mathbf{G}_{i}\right|=v_{i}$ is the weak Chern number [Fig. 1(b)] in each of the Brillouin-zone (BZ) planes normal to $\mathbf{R}_{i}$ [2-4,33-35] [36]. In Eq. (1), the axion angle $\theta$ governs the magnetoelectric response [4,5]; in terms of the non-Abelian Berry connection $\mathcal{A}$,

$$
\theta[\mathcal{A}]=\frac{1}{4 \pi} \int d^{3} k \epsilon^{i j k} \operatorname{tr}\left(\mathcal{A}_{i} \partial_{j} \mathcal{A}_{k}-\frac{2 i}{3} \mathcal{A}_{i} \mathcal{A}_{j} \mathcal{A}_{k}\right) .
$$

Spatial inversion $(\mathcal{I}$ ) and $\mathcal{T}$ symmetries (as well as other, more complicated symmetries) [28] act to quantize $\theta$ as a 

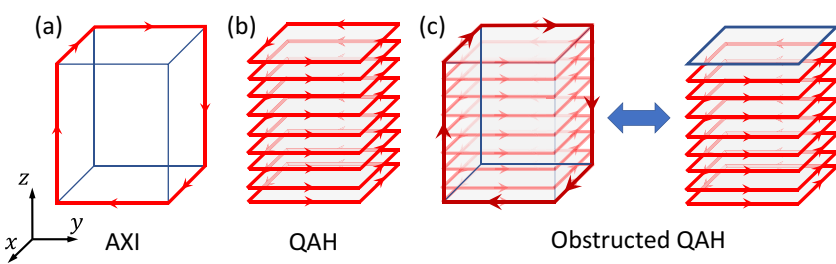

Obstructed QAH

FIG. 1. (a) An $\mathcal{I}$-symmetric AXI with $v_{x, y, z}=0, \theta=\pi$. (b) A $\hat{\mathbf{z}}$-directed, $\mathcal{I}$-symmetric weak stack of Chern insulators with a nontrivial QAH effect $\left[v_{z}=-1, v_{x}=v_{y}=\theta=0\right]$. (c) In a superposition of (a) and (b), the hinge states generically hybridize with the QAH surface states. Hence the superposition can be deformed into an oQAH insulator $\left[v_{z}=-1, v_{x}=v_{y}=0, \theta=\pi\right]$ equivalent to shifting the QAH insulator in (b) by a half-lattice translation. A finite-sized oQAH insulator exhibits either coexisting surface and hinge states or exactly one fewer (or one more) QAH surface state, depending on whether $\mathcal{I}$ symmetry is weakly broken.

$\mathbb{Z}_{2}$ topological invariant for which $\theta=0(\theta=\pi)$ is the trivial (topological) value [4,5,31] [37]. In particular, $v_{x, y, z}=0$, $\theta=\pi$ defines a 3D TI when $\mathcal{T}$ symmetry quantizes $\theta$ and defines a magnetic axion insulator (AXI) when $\mathcal{T}$ symmetry is absent and $\theta$ is instead quantized by $\mathcal{I}[4,5,27,28,38,39]$. In 3D TIs and AXIs, the combination of $\theta=\pi$ and $v_{x, y, z}=0$ leads to unusual response properties, including low-energy excitations resembling magnetic monopoles (the Witten effect $[40,41])$ and quantized Faraday and Kerr rotations [4,42]. AXIs have recently been recognized as "higher-order" TIs (HOTIs) [28-30,43-60] featuring gapped surfaces and odd numbers of sample-encircling chiral hinge modes [Fig. 1(a)]. AXI phases have been proposed in a number of compounds [43,61,62] and observed in Mn-doped 3D TIs [63-66].

A $\theta=\pi$ phase also appears in the low-energy field theory of a topological semimetal gapped by a chargedensity wave (CDW) distortion [1,2,67-69]. Specifically, in Weyl semimetals (WSMs) — whose bulk Fermi pockets [Weyl points (WPs)] are sources and sinks of Berry curvature characterized by integer-valued topological (chiral) charges [70-72] [Fig. 2(a)]—it was shown at the $\mathbf{k} \cdot \mathbf{p}$ level that $\theta(\mathbf{x}, t)=$ $\theta_{0}+\phi(\mathbf{x}, t)$, where $\theta_{0}=\mathbf{Q} \cdot \mathbf{x}$ is the contribution to $\theta(\mathbf{x}, t)$
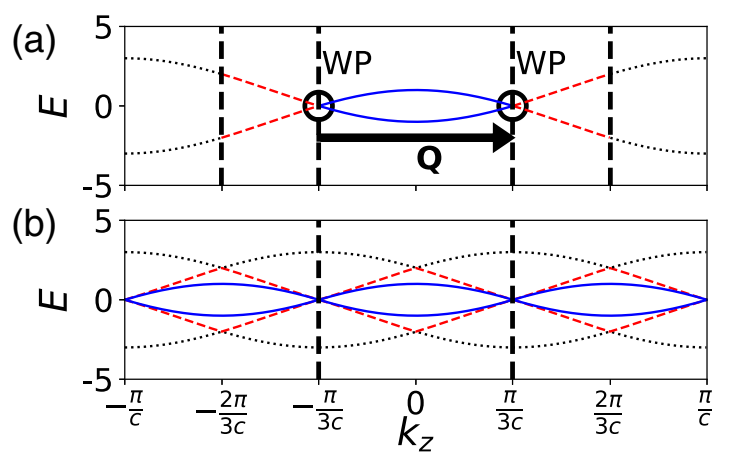

FIG. 2. BZ and band structure folding from a CDW with $\mathbf{Q}=$ $(2 \pi / N c) \hat{\mathbf{z}}, N=3$. (a) A minimal $\mathcal{I}$-symmetric WSM with two WPs at $k_{z}= \pm \pi / 3 c$ and a BZ with $\mathbf{G}_{z}=(2 \pi / c) \hat{\mathbf{z}}$ [Eq. (5)]. Solid blue (red dashed) [black dotted] bands lie in the first (second) [third] third of the first BZ. (b) The rBZ, for which $\mathbf{G}_{z}^{\prime}=\mathbf{Q}$. The WPs from (a) are folded in (b) into an (unstable) fourfold degeneracy at the rBZ boundary. from two WPs separated by a momentum $\mathbf{Q}$ and $\phi(\mathbf{x}, t)$ is the (dynamical) phase of the CDW order parameter [1,2,67-69]. The appearance of an axionic response was attributed to the chiral anomaly in quantum field theory. $\phi(\mathbf{x}, t)$ is a Goldstone mode and hence can be tuned freely; uniform shifts of $\phi(\mathbf{x}, t)$ are the current-carrying sliding mode of the CDW. In disordered or incommensurate CDWs, however, $\phi(\mathbf{x}, t)$ is typically pinned to a nonuniversal value [73-75]. References [1,2,6769] have recently been revisited in light of experiments on the CDW compound $\left(\mathrm{TaSe}_{4}\right)_{2} \mathrm{I}[76,77]$ demonstrating WPs at high temperatures $[78,79]$ and nonlinear negative magnetoresistance consistent with a gapped dynamical Weyl-CDW [80].

Confusingly, the dynamical Weyl-CDW is frequently labeled an AXI in the literature [1,2]. However, because the Weyl-CDW response in Refs. [1,2] was derived from a $\mathbf{k} \cdot \mathbf{p}$ approximation, and at static $\phi$ in Ref. [3], it remains an open and urgent question whether there exists a UV completion in which $\delta \theta_{\phi}=\theta_{\phi=\pi}-\theta_{\phi=0} \bmod 2 \pi=\pi$ emerges due to the topology of band electrons, and whether the bulk at $\phi=0, \pi$ is a single-particle AXI. In this Rapid Communication, we demonstrate that the $\mathcal{I}$-symmetric UV completion of the simplest dynamical Weyl-CDW is not an AXI, but is instead, depending on $\phi$, one of two topologically distinct QAH phases-a QAH insulator or an "obstructed" QAH (oQAH) insulator [Figs. 1(b) and 1(c)] — that differ by a fractional lattice translation. Crucially, although $\theta$ is origin dependent in the presence of a background QAH [4,30,54], we find that the QAH and oQAH phases still differ by an origin-(gauge-) independent, topological axion angle $\delta \theta_{\phi}=$ $\pi$ that reflects a difference in $\mathcal{I}$-quantized "Chern number polarization" $[54,81]$. This provides a direct analogy between axionic CDWs and the Su-Schrieffer-Heeger model of an $\mathcal{I}$-symmetric CDW in polyacetylene [82], in which both phases are trivial atomic limits that differ by a fractional lattice translation corresponding to an $\mathcal{I}$-quantized topological polarization. We demonstrate that the relative axionic response of two $\mathcal{I}$-symmetric Weyl-CDWs originates from their single-particle band topology. We generalize our findings to multiband systems with two WPs and to incommensurate CDWs, establishing that QAH insulators and topological phase shifts $\delta \theta_{\phi}=\pi$ are generic in minimal $\mathcal{I}$ symmetric Weyl-CDWs. Our focus on magnetic Weyl-CDWs is further justified by recent experiments demonstrating the existence of tunable magnetic WSM phases in $\mathrm{Co}_{2} \mathrm{MnGa}$ [83], $\mathrm{Co}_{3} \mathrm{Sn}_{2} \mathrm{~S}_{2}$ [84,85], $\mathrm{CoS}_{2}$ [86], CeAlGe [87], $\mathrm{Mn}_{3} \mathrm{Sn}[88,89]$, and $\mathrm{Mn}_{3} \mathrm{ZnC}$ [90], many of which host successive, symmetrylowering magnetic phase transitions below room temperature. In the conclusion and Supplemental Material (SM) [91], we provide additional models and analysis generalizing our results to nonmagnetic Weyl- and Dirac-CDWs.

To begin, we introduce a simple model of a $\mathcal{T}$-broken (magnetic), $\mathcal{I}$-symmetric WSM with two WPs and with orthorhombic lattice vectors of length $a, b, c$ in the $\hat{\mathbf{x}}, \hat{\mathbf{y}}, \hat{\mathbf{z}}$ directions, respectively [92]:

$$
\begin{aligned}
H(\mathbf{k})= & -2\left[t_{x} \sigma^{x} \sin \left(k_{x} a\right)-t_{y} \sigma^{y} \sin \left(k_{y} b\right)\right] \\
& +2 t_{z} \sigma^{z}\left[\cos \left(k_{z} c\right)-\cos \frac{Q c}{2}\right] \\
& -m \sigma^{z}\left[2-\cos \left(k_{x} a\right)-\cos \left(k_{y} b\right)\right],
\end{aligned}
$$


where $m / 2>t_{x}, t_{y}, t_{z}>0$. Equation (5) is gapped at half filling at all $\mathbf{k}$ points away from two WPs at $\mathbf{k}=(0,0, \pm Q / 2)$ with chiral charges $C= \pm 1$ [Fig. 2(a)] related by $\mathcal{I}$, here represented by $\mathcal{I} H(\mathbf{k}) \mathcal{I}^{-1}=\sigma^{z} H(-\mathbf{k}) \sigma^{z}$. As shown in Refs. [93,94] and in the Supplemental Material [91], the occupied parity $(\mathcal{I})$ eigenvalues imply the k-space Chern numbers $C_{z}\left(c k_{z}=0\right) \bmod 2=-1, C_{z}\left(c k_{z}=\pi\right) \bmod 2=0$, mandating the appearance of the $|C|=1$ WPs.

We next construct a $\mathbf{k} \cdot \mathbf{p}$ expansion of Eq. (5) about the two WPs:

$$
H(\mathbf{q}) \approx-\left(2 t_{x} a q_{x} \sigma^{x}-2 t_{y} b q_{y} \sigma^{y}\right) \tau^{0}+2 t_{z} c q_{z} \sin \frac{Q c}{2} \sigma^{z} \tau^{z},
$$

where the Pauli matrices $\vec{\tau}$ act in the space of electron annihilation operators $c_{1 / 2, \mathbf{k}}$ as

$$
c_{\mathbf{R}} \approx \sum_{\mathbf{k}} c_{1 \mathbf{k}} e^{i \mathbf{R} \cdot[(Q / 2) \hat{\mathbf{z}}+\mathbf{k}]}+c_{2 \mathbf{k}} e^{-i \mathbf{R} \cdot[(Q / 2) \hat{\mathbf{z}}-\mathbf{k}]} .
$$

Equation (6) can be gapped by a CDW distortion,

$$
\begin{aligned}
H_{\mathrm{CDW}} & =2 \sum_{\mathbf{R}}|\Delta| \cos \left(Q R_{z}+\phi\right) c_{\mathbf{R}}^{\dagger} \sigma^{z} c_{\mathbf{R}} \\
& =|\Delta| \sum_{\mathbf{k}}\left(c_{\mathbf{k}-\frac{Q}{2} \hat{\mathbf{z}}}^{\dagger} \sigma^{z} c_{\mathbf{k}+\frac{Q}{2} \hat{\mathbf{z}}} e^{-i \phi}+\text { H.c. }\right),
\end{aligned}
$$

which breaks the translation symmetry of Eq. (5), coupling the two WPs and inducing a mass in Eq. (6),

$$
V_{\phi}=|\Delta| \sigma^{z}\left(\tau^{x} \cos \phi-\tau^{y} \sin \phi\right),
$$

that opens a gap at all $\phi$ for $Q \neq \pi / c,|\Delta|>0$. Crucially, $\mathcal{I}$ symmetry is now represented in Eqs. (6) and (10) by $\mathcal{I} H(\mathbf{q}) \mathcal{I}^{-1}=\sigma^{z} \tau^{x} H(-\mathbf{q}) \sigma^{z} \tau^{x}$, such that Eq. (10) only preserves $\mathcal{I}$ (centered at the origin) for $\phi=0, \pi$ when $Q \neq \pi / c$ (see Ref. [91]).

Consistent with previous works [1,2,67-69], a domain wall between $\phi=0, \pi$ is equivalent to the critical point between a trivial insulator and an AXI [4,28]. Correspondingly, because $\left\{H(\mathbf{q}), V_{\phi}\right\}=0$ for all $\phi, \mathcal{I}$-breaking defects in the space $(\Delta, \phi)$ will bind chiral modes [95] (the axion strings in Ref. [1]). In Refs. [1,2,4,6,96], the authors used the chiral anomaly to motivate the appearance of chiral modes, identifying the relationship $\theta=[\pi / 2](1-\operatorname{sgn}[\cos \phi]) \bmod 2 \pi$ for $\phi=0, \pi . \delta \theta_{\phi}=\delta \phi$ is also consistent with magnetic symmetry-based indicators $\left\{\tilde{z}_{4} \mid \tilde{z}_{2 x} \tilde{z}_{2 y} \tilde{z}_{2 z}\right\}$ for $3 \mathrm{D}$ crystals with $\mathcal{I}$ and translation symmetries [28,43-45,48,50-52,57-59]:

$$
\begin{aligned}
\tilde{z}_{4} & =\frac{1}{2} \sum_{\mathbf{k}_{a} \in \text { TRIMS }}\left(n_{+}^{a}-n_{-}^{a}\right) \bmod 4, \\
\tilde{z}_{2, i} & =\frac{1}{2} \sum_{\mathbf{k}_{\mathbf{a}} \cdot \mathbf{R}_{\mathbf{i}}=\pi \in \mathrm{TRIMS}}\left(n_{+}^{a}-n_{-}^{a}\right) \bmod 2,
\end{aligned}
$$

where $n_{ \pm}^{a}$ are the number of valence \pm 1 parity eigenvalues at $\mathbf{k}_{a}$, and where TRIM is short for time-reversal-invariant momentum. Specifically, $\delta \phi=\pi$ in Eq. (10) implies that $\left|\delta \tilde{z}_{4}\right|=2$. However, because weak Chern numbers are only $\mathcal{I}$-symmetry-indicated modulo 2 [93,94], $\left|\delta \tilde{z}_{4}\right|=2$ does not itself indicate an AXI transition. Additionally, when $v_{z} \neq 0$, defining $\theta$ uniquely requires the specification of a reference state and $\mathcal{I}$ center (i.e., an origin) [54]. Furthermore, if there are other bulk or surface contributions to the topological response (e.g., other massive Dirac fermions at larger momenta, or a background QAH), then defects in $V_{\phi}$ will host additional states that coexist with and obscure the AXI bound states. Therefore, to fully determine the topology of the WeylCDW, we will analyze the lattice-regularized UV completion [Eqs. (5) and (9)] beyond Eq. (11).

When $Q=2 \pi / N c, N \in \mathbb{Z}^{+}$in Eq. (5), the CDW is lattice commensurate, and Eq. (5) remains periodic in a folded (reduced) BZ (rBZ) with $\mathbf{G}^{\prime}=Q \hat{\mathbf{z}}$ that includes bands translated from $\left|k_{z}\right|>\pi /(N c)$ [Fig. 2(b)]. For all values of $N$, the two WPs fold into a linear fourfold (Dirac) [97] degeneracy at the rBZ boundary [Eq. (6)]. We deduce from the bulk parity eigenvalues that $C_{z}\left(\left|k_{z} c\right|<\pi / N\right)=-1, C_{z}\left(\left|k_{z} c\right|>\pi / N\right)=$ 0 for all $N \in \mathbb{Z}^{+}$[98], implying that $v_{z}=-1$ in the $\mathrm{rBZ}$ [Fig. 2(b)], independent of whether $\phi=0, \pi$. Combining $v_{z}=-1$ with the $\mathbf{k} \cdot \mathbf{p}$ analysis preceding Eq. (11) and fixing the origin to $z=0$ in the modulated cell, we find that $\phi=0$ $[\phi=\pi]$ corresponds to a $\{2 \mid 001\} \hat{\mathbf{z}}$-directed weak Chern (i.e., QAH) insulator $[\{0 \mid 001\}$ oQAH insulator $]$ (see Fig. 1) with $v_{x, y}=0, v_{z}=-1$ and $\theta=0[\theta=\pi]$. Despite the QAH and oQAH insulators differing by a translation $(N c / 2) \hat{\mathbf{z}}, \delta \theta_{\phi}=\pi$, independent of the choice of origin. In the SM, we provide further details and analytically compute $v_{x, y, z}$ and $\delta \theta_{\phi}$ for $N=2$.

To explicitly determine the bulk topology, we will employ model-agnostic numerical methods $[28,30,54]$ to extract $v_{z}(\phi)$ and $\delta \theta_{\phi}$. To begin, we fix the origin to the $\mathcal{I}$ center at $(x, y, z)=(0,0,0)$ in the modulated cell and form an $\mathbf{R}_{i^{-}}$ directed, $\mathcal{I}$-symmetric slab. The Hall conductance $G_{H, i}$ of the slab consists of an extensive contribution from the bulk QAH and an intensive contribution from $\theta$ that reflects either the bulk magnetoelectric polarizability or a QAH effect offset from the origin $[4,30,54]$ :

$$
G_{H, i}=\sigma_{H, i} L_{i}+\left(e^{2} \theta / h \pi\right),
$$

where $\sigma_{H, i}=e^{2} \nu_{i} \mathbf{G}_{i} / 2 \pi h$ is the Hall conductivity (given by the weak Chern number $v_{i}$ ) and $L_{i}$ is the (lattice-regularized) thickness of the slab. Because a slab is a quasi-2D system, it carries a quantized Chern number $\mathcal{G}_{i}$ that is related to Eq. (12) by $\mathcal{G}_{i} e^{2} / h=G_{H, i}$. For $\mathcal{I}$-symmetric slab geometries, $\theta$ remains quantized to the bulk value and provides an odd-integer contribution to Eq. (12) when $\theta \bmod 2 \pi=\pi$ [28]—-this effect manifests in finite $3 \mathrm{D}$ systems with $v_{x, y, z}=0$ (i.e., AXIs) via chiral hinge modes [Fig. 1(a)]. Therefore, given a fixed, $\mathcal{I}$-symmetric, $\mathbf{R}_{i}$-directed slab, and knowledge of $v_{i}$, changes in $\theta$ can be numerically extracted through Eq. (12). For the Weyl-CDWs in this work, our choice of origin corresponds to a convention in which $\theta=0$ when $\mathcal{G}_{z}=v_{z} L_{z} /(N c)$ [99].

As an example, we analyze Eq. (5) with commensurate $Q=2 \pi / 3 c$. The tight-binding model in the rBZ exhibits the symmetry-based indicators $\{2 \mid 001\}(\{0 \mid 001\})$ at $\phi=0(\phi=$ $\pi$ ) [Eq. (11)] (see Ref. [91]). In Figs. 3(a) and 3(b), we plot the $\hat{\mathbf{y}}$-normal surface Green's function and spectrum, which are identical for $\phi=0, \pi$. Because surface Green's functions do not capture hinge states [26,28,55,100-102] [103] (or origindependent changes in $\theta$ ), the spectral flow in Fig. 3(b) and the flat band in Fig. 3(a) indicate a bulk QAH contribution $\nu_{z}(\phi=0, \pi)=-1$. 

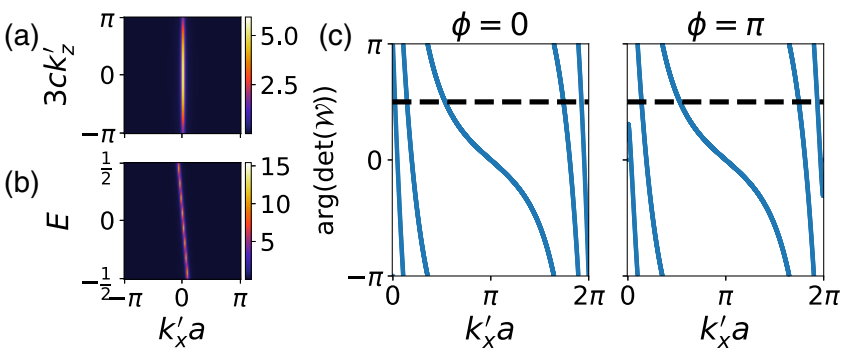

FIG. 3. Bulk $\left(v_{z}\right)$ and slab $\left(\mathcal{G}_{z}\right)$ Chern numbers for an $N=3$ $(Q=2 \pi / 3 c)$ commensurate CDW. (a) The $\hat{\mathbf{y}}$-surface spectral function at $E=0$ exhibits a flat band. (b) The $\hat{\mathbf{y}}$-surface spectral function at $k_{z}^{\prime}=0$ exhibits $C_{z}\left(3 k_{z}^{\prime} c\right)=-1$ spectral flow along $k_{x}^{\prime}$. The spectral functions in (a) and (b) are the same for $\phi=0, \pi$. (c) The $\hat{\mathbf{y}}$-directed slab Berry phase [28] for a slab with five unit cells in the $\hat{\mathbf{z}}$ direction exhibits $\mathcal{G}_{z}=-5\left(\mathcal{G}_{z}=-4\right)$ spectral flow for $\phi=0(\phi=\pi)$. (a)(c) imply that the Weyl-CDW at $\phi=0(\phi=\pi)$ is a QAH (oQAH) insulator [Figs. 1(b) and 1(c)] and that $\delta \theta_{\phi}=\pi$.

We next cut the model in Figs. 3(a) and 3(b) into an $\mathcal{I}$-symmetric, $\hat{\mathbf{z}}$-directed slab geometry with $L_{z} / 3 c=5$ unit cells and calculate the $\hat{\mathbf{y}}$-directed non-Abelian slab Berry phase (Wilson loop) $\mathcal{W}[28,104-106]$ [Fig. 3(c)], whose winding indicates that $\mathcal{G}_{z}(0)=-5=v_{z} L_{z} / 3 c, \mathcal{G}_{z}(\pi)=-4=$ $v_{z} L_{z} / 3 c+1$. Along with $v_{z}(\phi=0, \pi)=-1,\left|\delta \mathcal{G}_{z}\right|=1$ indicates through Eq. (12) that the insulating Weyl-CDW at $\phi=0$ $(\phi=\pi)$ is a QAH (oQAH) insulator, implying that $\delta \theta_{\phi}=\pi$.

Having demonstrated that $\mathcal{I}$-symmetric, commensurate Weyl-CDWs are either QAH or oQAH insulators, we next explore the case of incommensurate modulation. Although an incommensurate CDW is not translationally invariant, neither QAH nor oQAH phases require translation symmetry $[4,5,27,28,38,39]$. Consequently, Eq. (12) still applies, without modification, to $\hat{\mathbf{z}}$-directed slabs of Eq. (5) with incommensurate values of $Q$.

To confirm this result, we first cut $H_{0}+H_{\text {CDw }}$ [Eqs. (5) and (9)] with $Q=\varphi \pi / 2 c$ (where $\varphi$ is the golden ratio) into an $\mathcal{I}$-symmetric rod geometry. We observe an extensive number of QAH surface states along the rod for $\phi=0, \pi$, where there is exactly one fewer surface chiral mode at $\phi=\pi$ [Fig. 4(a)]. Next, to measure $\theta$, we cut the incommensurate Weyl-CDW into an $\mathcal{I}$-symmetric, $\hat{\mathbf{z}}$-directed slab and calculate the $\hat{\mathbf{y}}$-directed Berry phase, as we previously did in Fig. 3(c). In the slab geometry, $\left|\delta \mathcal{G}_{z}\right|=1$ between $\phi=0, \pi$ [Fig. 4(b)]. Furthermore, in incommensurate CDWs, tuning $\phi$ changes the bulk wave functions, but not the bulk energy spectrum $[107,108]$, such that a bulk-insulating, incommensurate Weyl-CDW with $\phi=0$ remains insulating at arbitrary $\phi$. Additionally, $v_{z}$ cannot change without a bulk gap closure, whereas $\theta$ is free to wind between 0 and $\pi$ at $\mathcal{I}$-breaking CDW angles away from $\phi=0, \pi[4,5,28]$. As shown in the $\mathrm{SM},\left|\delta \mathcal{G}_{z}\right|=1$ in Fig. 4(b), along with $G_{H, z}$ calculated for successive rational appoximants of an irrational $Q$, imply that, as in the commensurate case (Fig. 3), the incommensurate Weyl-CDW carries the relative axion angle $\delta \theta_{\phi}=\pi$.

Our results have several implications for experimental investigations of axionic response in Weyl-CDWs. First, we have demonstrated that a large QAH effect is unavoidable
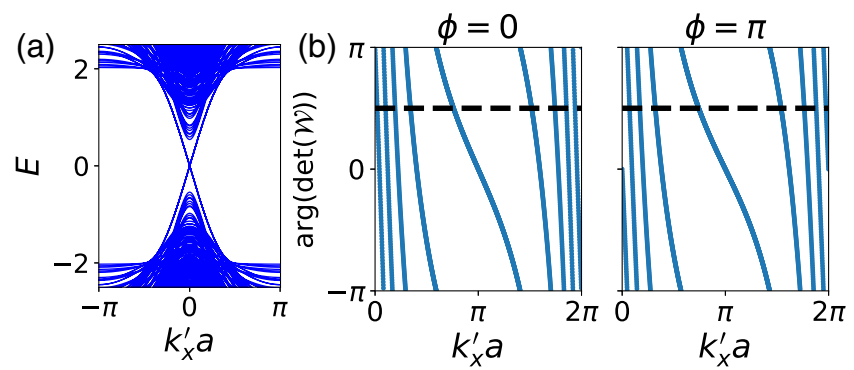

FIG. 4. (a) Band structure for the incommensurate Weyl-CDW in an $\mathcal{I}$-symmetric, $\hat{\mathbf{x}}$-directed rod geometry with 21 sites in the $\hat{\mathbf{y}}$ and $\hat{\mathbf{z}}$ directions and with $\phi=0$. The chiral states traversing the gap have degeneracy proportional to the rod thickness, indicating that they are QAH background surface states. When $\phi=\pi$, the rod spectrum is qualitatively the same as (a), but exhibits one fewer pairs of chiral modes. (b) The $\hat{\mathbf{y}}$-directed Wilson loop of an $\mathcal{I}$-symmetric, $\hat{\mathbf{z}}$-directed slab [28] of the model in (a) with 21 layers exhibits $\mathcal{G}_{z}=-9\left(\mathcal{G}_{z}=\right.$ -8) spectral flow for $\phi=0(\phi=\pi)$, implying that $\delta \theta_{\phi}=\pi$.

and guaranteed in both commensurate and incommensurate minimal Weyl-CDWs, independent of $\phi$. Second, the interplay between lattice and phase-angle defects, which both bind 1D chiral modes, is a fruitful area for future study, though one must cautiously separate contributions from $\theta$ and those from a background QAH effect $[109,110]$. Next, we emphasize that the axionic response in Weyl-CDWs is measurable through the dynamical dependence of the quasi-2D QAH effect on $\phi$, rather than through the static magnetoelectric polarizability at fixed $\phi[111,112]$. Furthermore, solitonlike defects in $\phi$, which carry the same half-quantized Hall conductivity as gapped AXI surfaces for $\delta \theta_{\phi}=\pi$ [113], can in principle be manipulated by exciting the CDW sliding mode. Finally, in $\mathcal{I}$-symmetric, magnetic Weyl-CDWs, our results highlight the experimental and theoretical difficulty of distinguishing QAH, oQAH, and AXI phases. However, our results do imply that by carefully computing $\mathbf{k}$-space Chern numbers and then zone folding, it is possible to predict the topology of Weyl-CDWs in real materials without performing intensive quasiperiodic calculations.

Our methodology can be extended to spin-density waves [69,114-116] and CDWs in $\mathcal{T}$-symmetric semimetals, including Dirac [97,117,118], Weyl, and nodal-line semimetals [119-122], which also exhibit signatures of higher-order topology $[55,100,123,124]$. Most interestingly, because rotation- and $\mathcal{T}$-symmetric HOTIs $[28,56]$ can be formed from weak stacks of 2D TIs [48,51], rotationsymmetric CDWs in $\mathcal{T}$-symmetric WSMs, such as the CDW in $\left(\mathrm{TaSe}_{4}\right)_{2} \mathrm{I}$ [78], may also exhibit nontrivial response effects. Specifically, a CDW can fold four WPs in a rotation- and $\mathcal{T}$ symmetric WSM into an eightfold double Dirac point (DDP) in which line defects bind helical modes equivalent to HOTI hinge states $[125,126]$; alternatively, a DDP critical point can also be realized by coupling two fourfold Dirac points with a CDW. Recent experiments have demonstrated hinge-statelike step-edge helical modes and robust edge supercurrents in rotation- and $\mathcal{T}$-symmetric WSMs [127-131], as well as a stable DDP and topological step-edge modes in the CDW phase of $\mathrm{TaTe}_{4}$ [132]. In the SM, we present an explicit 
model demonstrating that a $\mathcal{T}$-symmetric Dirac-CDW hosts an eightfold DDP critical point that separates weak TI (WTI) and "obstructed" WTI (oWTI) phases that differ by a helical HOTI. Unlike in the QAH and oQAH Weyl-CDW phases, the difference between the WTI and oWTI Dirac-CDWs cannot be connected to a known response theory, because a $\theta$-like topological field theory for helical HOTIs has not yet been elucidated $[28,45,55]$, suggesting an intriguing direction for future study.

Note added. Recently, Ref. [133] also demonstrated that minimal $\mathcal{T}$-symmetric Weyl-CDWs are topologically equivalent to $\phi$-dependent weak TIs-the results of Ref. [133] are complementary to and in complete agreement with the results of this work. Recently, a stable DDP and topological stepedge modes were experimentally measured in the CDW phase of the $\mathcal{T}$-symmetric Dirac semimetal $\mathrm{TaTe}_{4}$ [132], providing further support for the analysis performed in this work. Additionally, recently, an analysis of minimal Weyl-CDWs beyond mean-field theory was performed in [113]; the analysis in [113] explicitly confirms our characterization of the mean-field QAH and oQAH phases of Weyl-CDWs. Lastly, recently, Ref. [134] also demonstrated a relationship between AXI pumping cycles and hybrid-Wannier-sheet flow that is equivalent to the Weyl-CDWs studied in this work when the CDW angle $\phi$ is treated as a dynamical parameter.

We acknowledge helpful conversations with Peter Abbamonte, B. Andrei Bernevig, Matthew Gilbert, Chao-Xing Liu, and Jiabin Yu, as well as crucial input from Zhida Song on an early version of this work. B.J.W. acknowledges support from B. Andrei Bernevig through Department of Energy Grant No. DE-SC0016239, Simons Investigator Grant No. 404513, U.S.-Israel Binational Science Foundation Grant No. 2018226, and ONR Grant No. N00014-20-1-2303. B.B. acknowledges the support of the Alfred P. Sloan Foundation and National Science Foundation Grant No. DMR-1945058.
[1] Z. Wang and S.-C. Zhang, Phys. Rev. B 87, 161107(R) (2013).

[2] Y. You, G. Y. Cho, and T. L. Hughes, Phys. Rev. B 94, 085102 (2016).

[3] M. Laubach, C. Platt, R. Thomale, T. Neupert, and S. Rachel, Phys. Rev. B 94, 241102(R) (2016).

[4] X.-L. Qi, T. L. Hughes, and S.-C. Zhang, Phys. Rev. B 78, 195424 (2008).

[5] A. M. Essin, J. E. Moore, and D. Vanderbilt, Phys. Rev. Lett. 102, 146805 (2009).

[6] F. Wilczek, Phys. Rev. Lett. 58, 1799 (1987).

[7] S. C. Zhang, T. H. Hansson, and S. Kivelson, Phys. Rev. Lett. 62, 82 (1989).

[8] B. I. Halperin, P. A. Lee, and N. Read, Phys. Rev. B 47, 7312 (1993).

[9] D. T. Son, Phys. Rev. X 5, 031027 (2015).

[10] D. P. DiVincenzo and E. J. Mele, Phys. Rev. B 29, 1685 (1984).

[11] G. W. Semenoff, Phys. Rev. Lett. 53, 2449 (1984).

[12] A. H. Castro Neto, F. Guinea, N. M. R. Peres, K. S. Novoselov, and A. K. Geim, Rev. Mod. Phys. 81, 109 (2009).

[13] L. Fu, C. L. Kane, and E. J. Mele, Phys. Rev. Lett. 98, 106803 (2007).

[14] L. Fu and C. L. Kane, Phys. Rev. B 76, 045302 (2007).

[15] D. Hsieh, D. Qian, L. Wray, Y. Xia, Y. S. Hor, R. J. Cava, and M. Z. Hasan, Nature (London) 452, 970 (2008).

[16] Y. Xia, D. Qian, D. Hsieh, L. Wray, A. Pal, H. Lin, A. Bansil, D. Grauer, Y. S. Hor, R. J. Cava, and M. Z. Hasan, Nat. Phys. 5, 398 (2009).

[17] M. F. Lapa, Phys. Rev. B 99, 235144 (2019).

[18] F. D. M. Haldane, Phys. Rev. Lett. 61, 2015 (1988).

[19] B. Bernevig and T. Hughes, Topological Insulators and Topological Superconductors (Princeton University Press, Princeton, NJ, 2013).

[20] M. Z. Hasan and C. L. Kane, Rev. Mod. Phys. 82, 3045 (2010).

[21] E. Fradkin, E. Dagotto, and D. Boyanovsky, Phys. Rev. Lett. 57, 2967 (1986).

[22] L. Alvarez-Gaumé and E. Witten, Nucl. Phys. B 234, 269 (1984).

[23] A. N. Redlich, Phys. Rev. Lett. 52, 18 (1984).
[24] R. Jackiw, Phys. Rev. D 29, 2375 (1984).

[25] M. Mulligan and F. J. Burnell, Phys. Rev. B 88, 085104 (2013).

[26] B. J. Wieder, B. Bradlyn, Z. Wang, J. Cano, Y. Kim, H.-S. D. Kim, A. M. Rappe, C. L. Kane, and B. A. Bernevig, Science 361, 246 (2018).

[27] T. L. Hughes, E. Prodan, and B. A. Bernevig, Phys. Rev. B 83, 245132 (2011).

[28] B. J. Wieder and B. A. Bernevig, arXiv:1810.02373.

[29] F. Zhang, C. L. Kane, and E. J. Mele, Phys. Rev. Lett. 110, 046404 (2013).

[30] N. Varnava and D. Vanderbilt, Phys. Rev. B 98, 245117 (2018).

[31] E. Witten, Rev. Mod. Phys. 88, 035001 (2016).

[32] In this work we will use greek indices as space-time indices and roman indices as purely spatial indices.

[33] B. I. Halperin, Jpn. J. Appl. Phys. 26, 1913 (1987).

[34] M. Kohmoto, B. I. Halperin, and Y.-S. Wu, Phys. Rev. B 45, 13488 (1992).

[35] F. D. M. Haldane, Phys. Rev. Lett. 93, 206602 (2004).

[36] Note that for gapless systems, such as Weyl semimetals, $C_{i}(\mathbf{k}$. $\mathbf{R}_{i}$ ) will instead depend on $\mathbf{k}$.

[37] We note that because Eq. (1) is an action, only $\theta \bmod 2 \pi$ is a gauge-invariant quantity. This is reflected in the gauge ambiguity of the Chern-Simons term [Eq. (4)].

[38] A. M. Turner, Y. Zhang, R. S. K. Mong, and A. Vishwanath, Phys. Rev. B 85, 165120 (2012).

[39] R. S. K. Mong, A. M. Essin, and J. E. Moore, Phys. Rev. B 81, 245209 (2010).

[40] E. Witten, Phys. Lett. B 86, 283 (1979).

[41] G. Rosenberg and M. Franz, Phys. Rev. B 82, 035105 (2010).

[42] L. Wu, M. Salehi, N. Koirala, J. Moon, S. Oh, and N. P. Armitage, Science 354, 1124 (2016).

[43] Y. Xu, L. Elcoro, Z. Song, B. J. Wieder, M. G. Vergniory, N. Regnault, Y. Chen, C. Felser, and B. A. Bernevig, arXiv:2003.00012.

[44] B. Bradlyn, L. Elcoro, J. Cano, M. G. Vergniory, Z. Wang, C. Felser, M. I. Aroyo, and B. A. Bernevig, Nature (London) 547, 298 (2017). 
[45] L. Elcoro, B. J. Wieder, Z. Song, Y. Xu, N. Regnault, B. Bradlyn, and B. A. Bernevig (unpublished).

[46] W. A. Benalcazar, B. A. Bernevig, and T. L. Hughes, Science 357, 61 (2017).

[47] W. A. Benalcazar, B. A. Bernevig, and T. L. Hughes, Phys. Rev. B 96, 245115 (2017).

[48] H. C. Po, A. Vishwanath, and H. Watanabe, Nat. Commun. 8, 50 (2017)

[49] J. Langbehn, Y. Peng, L. Trifunovic, F. von Oppen, and P. W. Brouwer, Phys. Rev. Lett. 119, 246401 (2017).

[50] H. Watanabe, H. C. Po, and A. Vishwanath, Sci. Adv. 4, eaat8685 (2018).

[51] E. Khalaf, H. C. Po, A. Vishwanath, and H. Watanabe, Phys. Rev. X 8, 031070 (2018).

[52] E. Khalaf, Phys. Rev. B 97, 205136 (2018).

[53] J. Ahn and B.-J. Yang, Phys. Rev. B 99, 235125 (2019).

[54] N. Varnava, I. Souza, and D. Vanderbilt, Phys. Rev. B 101, 155130 (2020).

[55] Z. Wang, B. J. Wieder, J. Li, B. Yan, and B. A. Bernevig, Phys. Rev. Lett. 123, 186401 (2019).

[56] C. Fang and L. Fu, Sci. Adv. 5, eaat2374 (2019).

[57] J. Yu, Z.-D. Song, and C.-X. Liu, Phys. Rev. Lett. 125, 036401 (2020).

[58] H. Kim, K. Shiozaki, and S. Murakami, Phys. Rev. B 100, 165202 (2019).

[59] R. Takahashi, Y. Tanaka, and S. Murakami, Phys. Rev. Research 2, 013300 (2020).

[60] O. Pozo, C. Repellin, and A. G. Grushin, Phys. Rev. Lett. 123, 247401 (2019)

[61] Y. Xu, Z. Song, Z. Wang, H. Weng, and X. Dai, Phys. Rev. Lett. 122, 256402 (2019).

[62] R.-X. Zhang, F. Wu, and S. Das Sarma, Phys. Rev. Lett. 124, 136407 (2020).

[63] M. M. Otrokov, I. I. Klimovskikh, H. Bentmann, D. Estyunin, A. Zeugner, Z. S. Aliev, S. Gaß, A. U. B. Wolter, A. V. Koroleva, A. M. Shikin, M. Blanco-Rey, M. Hoffmann, I. P. Rusinov, A. Y. Vyazovskaya, S. V. Eremeev, Y. M. Koroteev, V. M. Kuznetsov, F. Freyse, J. Sánchez-Barriga, I. R. Amiraslanov et al., Nature (London) 576, 416 (2019).

[64] E. D. L. Rienks, S. Wimmer, J. Sánchez-Barriga, O. Caha, P. S. Mandal, J. Ruzicka, A. Ney, H. Steiner, V. V. Volobuev, H. Groiss, M. Albu, G. Kothleitner, J. Michalicka, S. A. Khan, J. Minár, H. Ebert, G. Bauer, F. Freyse, A. Varykhalov, O. Rader et al., Nature (London) 576, 423 (2019).

[65] C. Liu, Y. Wang, H. Li, Y. Wu, Y. Li, J. Li, K. He, Y. Xu, J. Zhang, and Y. Wang, Nat. Mater. 19, 522 (2020).

[66] N. H. Jo, L.-L. Wang, R.-J. Slager, J. Yan, Y. Wu, K. Lee, B. Schrunk, A. Vishwanath, and A. Kaminski, Phys. Rev. B 102, 045130 (2020).

[67] A. A. Zyuzin and A. A. Burkov, Phys. Rev. B 86, 115133 (2012).

[68] A. A. Zyuzin, S. Wu, and A. A. Burkov, Phys. Rev. B 85, 165110 (2012).

[69] J. Maciejko and R. Nandkishore, Phys. Rev. B 90, 035126 (2014).

[70] X. Wan, A. M. Turner, A. Vishwanath, and S. Y. Savrasov, Phys. Rev. B 83, 205101 (2011).

[71] A. A. Burkov and L. Balents, Phys. Rev. Lett. 107, 127205 (2011).
[72] N. P. Armitage, E. J. Mele, and A. Vishwanath, Rev. Mod. Phys. 90, 015001 (2018).

[73] H. Fukuyama and P. A. Lee, Phys. Rev. B 17, 535 (1978).

[74] J. Bardeen, Phys. Rev. Lett. 45, 1978 (1980).

[75] G. Grüner, Rev. Mod. Phys. 60, 1129 (1988).

[76] P. Gressier, L. Guemas, and A. Meerschaut, Acta Crystallogr. Sect. B 38, 2877 (1982).

[77] R. J. Cava, P. Littlewood, R. M. Fleming, R. G. Dunn, and E. A. Rietman, Phys. Rev. B 33, 2439 (1986).

[78] W. Shi, B. J. Wieder, H. L. Meyerheim, Y. Sun, Y. Zhang, Y. Li, L. Shen, Y. Qi, L. Yang, J. Jena, P. Werner, K. Koepernik, S. Parkin, Y. Chen, C. Felser, B. A. Bernevig, and Z. Wang, arXiv:1909.04037.

[79] X.-P. Li, K. Deng, B. Fu, Y. Li, D. Ma, J. Han, J. Zhou, S. Zhou, and Y. Yao, arXiv:1909.12178.

[80] J. Gooth, B. Bradlyn, S. Honnali, C. Schindler, N. Kumar, J. Noky, Y. Qi, C. Shekhar, Y. Sun, Z. Wang, B. A. Bernevig, and C. Felser, Nature (London) 575, 315 (2019).

[81] O. Zilberberg, S. Huang, J. Guglielmon, M. Wang, K. P. Chen, Y. E. Kraus, and M. C. Rechtsman, Nature (London) 553, 59 (2018).

[82] W. P. Su, J. R. Schrieffer, and A. J. Heeger, Phys. Rev. Lett. 42, 1698 (1979).

[83] I. Belopolski, K. Manna, D. S. Sanchez, G. Chang, B. Ernst, J. Yin, S. S. Zhang, T. Cochran, N. Shumiya, H. Zheng, B. Singh, G. Bian, D. Multer, M. Litskevich, X. Zhou, S.-M. Huang, B. Wang, T.-R. Chang, S.-Y. Xu, A. Bansil et al., Science 365, 1278 (2019).

[84] D. F. Liu, A. J. Liang, E. K. Liu, Q. N. Xu, Y. W. Li, C. Chen, D. Pei, W. J. Shi, S. K. Mo, P. Dudin, T. Kim, C. Cacho, G. Li, Y. Sun, L. X. Yang, Z. K. Liu, S. S. P. Parkin, C. Felser, and Y. L. Chen, Science 365, 1282 (2019).

[85] N. Morali, R. Batabyal, P. K. Nag, E. Liu, Q. Xu, Y. Sun, B. Yan, C. Felser, N. Avraham, and H. Beidenkopf, Science 365, 1286 (2019).

[86] N. B. M. Schröter, I. Robredo, S. Klemenz, R. J. Kirby, J. A. Krieger, D. Pei, T. Yu, S. Stolz, T. Schmitt, P. Dudin, T. K. Kim, C. Cacho, A. Schnyder, A. Bergara, V. N. Strocov, F. de Juan, M. G. Vergniory, and L. M. Schoop, arXiv:2006.01557.

[87] P. Puphal, V. Pomjakushin, N. Kanazawa, V. Ukleev, D. J. Gawryluk, J. Ma, M. Naamneh, N. C. Plumb, L. Keller, R. Cubitt, E. Pomjakushina, and J. S. White, Phys. Rev. Lett. 124, 017202 (2020).

[88] K. Kuroda, T. Tomita, M.-T. Suzuki, C. Bareille, A. Â. A. Nugroho, P. Goswami, M. Ochi, M. Ikhlas, M. Nakayama, S. Akebi, R. Noguchi, R. Ishii, N. Inami, K. Ono, H. Kumigashira, A. Varykhalov, T. Muro, T. Koretsune, R. Arita, S. Shin et al., Nat. Mater. 16, 1090 (2017).

[89] N. H. Sung, F. Ronning, J. D. Thompson, and E. D. Bauer, Appl. Phys. Lett. 112, 132406 (2018).

[90] S. M. L. Teicher, I. K. Svenningsson, L. M. Schoop, and R. Seshadri, APL Mater. 7, 121104 (2019).

[91] See Supplemental Material at http://link.aps.org/ supplemental/10.1103/PhysRevResearch.2.042010 for a detailed exploration of models of Weyl- and Dirac-CDWs.

[92] T. M. McCormick, I. Kimchi, and N. Trivedi, Phys. Rev. B 95, 075133 (2017).

[93] X.-L. Qi, Y.-S. Wu, and S.-C. Zhang, Phys. Rev. B 74, 085308 (2006). 
[94] C. Fang, M. J. Gilbert, and B. A. Bernevig, Phys. Rev. B 86, 115112 (2012).

[95] J. C. Y. Teo and C. L. Kane, Phys. Rev. B 82, 115120 (2010).

[96] S. Ryu, A. P. Schnyder, A. Furusaki, and A. W. W. Ludwig, New J. Phys. 12, 065010 (2010).

[97] S. M. Young, S. Zaheer, J. C. Y. Teo, C. L. Kane, E. J. Mele, and A. M. Rappe, Phys. Rev. Lett. 108, 140405 (2012).

[98] Throughout this work, we choose parameters for which $v_{z}=-1$.

[99] This convention relies on $N$ being odd to avoid slabs with fractional modulated cells. In the SM, we consider a Weyl-CDW with $N=2$, which can only be cut into an $\mathcal{I}$-symmetric slab with a half-integer number of modulated cells.

[100] B. J. Wieder, Z. Wang, J. Cano, X. Dai, L. M. Schoop, B. Bradlyn, and B. A. Bernevig, Nat. Commun. 11, 627 (2020).

[101] F. Schindler, A. M. Cook, M. G. Vergniory, Z. Wang, S. S. P. Parkin, B. A. Bernevig, and T. Neupert, Sci. Adv. 4, eaat0346 (2018).

[102] F. Schindler, Z. Wang, M. G. Vergniory, A. M. Cook, A. Murani, S. Sengupta, A. Y. Kasumov, R. Deblock, S. Jeon, I. Drozdov, H. Bouchiat, S. Guéron, A. Yazdani, B. A. Bernevig, and T. Neupert, Nat. Phys. 14, 918 (2018).

[103] Specifically, surface Green's functions capture 2D degeneracies (e.g., surface Dirac cones and weak QAH chiral modes) that are protected by the symmetries of the $2 \mathrm{D}$ wallpaper groups [26]. Conversely, 1D hinge states are protected by the lower-symmetry quasi-1D frieze groups [45,101,121,135]. When a higher-order TI hinge with a particular Miller index is smoothed into a surface, the surface Green's function will hence only detect surface states that can be stabilized by the symmetries of the exposed surface [26,101,136,137]. Conversely, hinge states can be detected in hinge Green's function calculations, as employed in Refs. [61,100].

[104] L. Fidkowski, T. S. Jackson, and I. Klich, Phys. Rev. Lett. 107, 036601 (2011)

[105] R. Yu, X. L. Qi, A. Bernevig, Z. Fang, and X. Dai, Phys. Rev. B 84, 075119 (2011).

[106] A. Alexandradinata and B. A. Bernevig, Phys. Rev. B 93, 205104 (2016)

[107] Y. E. Kraus, Y. Lahini, Z. Ringel, M. Verbin, and O. Zilberberg, Phys. Rev. Lett. 109, 106402 (2012).

[108] C. de Lange and T. Janssen, Phys. Rev. B 28, 195 (1983).

[109] R. Queiroz, I. C. Fulga, N. Avraham, H. Beidenkopf, and J. Cano, Phys. Rev. Lett. 123, 266802 (2019).

[110] F. Schindler, S. S. Tsirkin, T. Neupert, B. A. Bernevig, and B. J. Wieder (unpublished).

[111] N. P. Armitage and L. Wu, SciPost Phys. 6, 46 (2019).

[112] K. S. Lin and B. Bradlyn (unpublished).

[113] D. Sehayek, M. Thakurathi, and A. A. Burkov, Phys. Rev. B 102, 115159 (2020).

[114] B. Roy, P. Goswami, and V. Juričić, Phys. Rev. B 95, 201102 (2017).

[115] G. Lemut, A. Donís Vela, M. J. Pacholski, J. Tworzydło, and C. W. J. Beenakker, New J. Phys. 22, 093022 (2020).
[116] N. Mitsuishi, Y. Sugita, M. S. Bahramy, M. Kamitani, T. Sonobe, M. Sakano, T. Shimojima, H. Takahashi, H. Sakai, K. Horiba, H. Kumigashira, K. Taguchi, K. Miyamoto, T. Okuda, S. Ishiwata, Y. Motome, and K. Ishizaka, Nat. Commun. 11, 2466 (2020).

[117] Z. Wang, H. Weng, Q. Wu, X. Dai, and Z. Fang, Phys. Rev. B 88, 125427 (2013).

[118] Z. Wang, Y. Sun, X.-Q. Chen, C. Franchini, G. Xu, H. Weng, X. Dai, and Z. Fang, Phys. Rev. B 85, 195320 (2012).

[119] Y. Kim, B. J. Wieder, C. L. Kane, and A. M. Rappe, Phys. Rev. Lett. 115, 036806 (2015).

[120] C. Fang, Y. Chen, H.-Y. Kee, and L. Fu, Phys. Rev. B 92, 081201(R) (2015).

[121] B. J. Wieder and C. L. Kane, Phys. Rev. B 94, 155108 (2016).

[122] L. M. Schoop, M. N. Ali, C. Straßer, A. Topp, A. Varykhalov, D. Marchenko, V. Duppel, S. S. P. Parkin, B. V. Lotsch, and C. R. Ast, Nat. Commun. 7, 11696 (2016).

[123] M. Lin and T. L. Hughes, Phys. Rev. B 98, 241103(R) (2018).

[124] C.-Z. Li, A.-Q. Wang, C. Li, W.-Z. Zheng, A. Brinkman, D.-P. Yu, and Z.-M. Liao, Phys. Rev. Lett. 124, 156601 (2020).

[125] B. J. Wieder, Y. Kim, A. M. Rappe, and C. L. Kane, Phys. Rev. Lett. 116, 186402 (2016).

[126] B. Bradlyn, J. Cano, Z. Wang, M. G. Vergniory, C. Felser, R. J. Cava, and B. A. Bernevig, Science 353, aaf5037 (2016).

[127] F.-T. Huang, S. Joon Lim, S. Singh, J. Kim, L. Zhang, J.W. Kim, M.-W. Chu, K. M. Rabe, D. Vanderbilt, and S.-W. Cheong, Nat. Commun. 10, 4211 (2019).

[128] Y.-B. Choi, Y. Xie, C.-Z. Chen, J. Park, S.-B. Song, J. Yoon, B. J. Kim, T. Taniguchi, K. Watanabe, J. Kim, K. C. Fong, M. N. Ali, K. T. Law, and G.-H. Lee, Nat. Mater. 19, 974 (2020),

[129] A. Kononov, G. Abulizi, K. Qu, J. Yan, D. Mandrus, K. Watanabe, T. Taniguchi, and C. Schönenberger, Nano Lett. 20, 4228 (2020).

[130] W. Wang, S. Kim, M. Liu, F. A. Cevallos, R. J. Cava, and N. P. Ong, Science 368, 534 (2020).

[131] I-L. Liu, C. Heikes, T. Yildirim, C. Eckberg, T. Metz, H. Kim, S. Ran, W. D. Ratcliff, J. Paglione, and N. P. Butch, npj Quantum Mater. 5, 62 (2020).

[132] X. Zhang, Q. Gu, H. Sun, T. Luo, Y. Liu, Y. Chen, Z. Shao, Z. Zhang, S. Li, Y. Sun, Y. Li, X. Li, S. Xue, J. Ge, Y. Xing, R. Comin, Z. Zhu, P. Gao, B. Yan, J. Feng et al., Phys. Rev. B 102, 035125 (2020).

[133] J. Yu, B. J. Wieder, and C.-X. Liu, arXiv:2008.10620.

[134] T. Olsen, T. Rauch, D. Vanderbilt, and I. Souza, Phys. Rev. B 102, 035166 (2020).

[135] J. Conway, H. Burgiel, and C. Goodman-Strauss, The Symmetries of Things (Taylor \& Francis, London, 2008).

[136] Z. Song, T. Zhang, Z. Fang, and C. Fang, Nat. Commun. 9, 3530 (2018).

[137] C.-H. Hsu, X. Zhou, T.-R. Chang, Q. Ma, N. Gedik, A. Bansil, S.-Y. Xu, H. Lin, and L. Fu, Proc. Natl. Acad. Sci. U.S.A. 116, 13255 (2019). 